\title{
ARTÍCULO DE REVISIÓN \\ Enfoque del tromboembolismo pulmonar agudo desde el momento inicial en el servicio de urgencias
}

Fecha de recibido:

19 de octubre de 2020.

Fecha de aprobación: 29 de enero de 2021
Forma de citar este artículo: Restrepo L, Ramírez A, Pérez $M$, Zuluaga JP, Moncayo JG. Enfoque del tromboembolismo pulmonar agudo desde el momento inicial en el servicio de urgencias. Med UPB. 2021;40(2):41-59. DOI:10.18566/medupb.v40n2.a07

1 Facultad de Medicina, Universidad CES. Medellín, Colombia.

2 Residencia de Anestesia, Universidad CES. Medellín, Colombia.

3 Clínica CES. Medellín, Colombia.

\section{Dirección de}

correspondencia: Laura

Restrepo Álvarez.

Correo electrónico:

laurarestrepoalv@gmail. com

\author{
Initial Approach to Acute Pulmonary Embolism since the Initial \\ Moment in the Emergency Department / Abordagem para \\ tromboembolismo pulmonar agudo desde o momento inicial \\ no departamento de emergência
}

Laura Restrepo Álvarez ${ }^{1}$, Andrés Ramírez Vélez², Marcela Pérez Muñoz ${ }^{1}$, Juan Pablo Zuluaga García ${ }^{1}$, Jairo Giovanni Moncayo Viveros ${ }^{3}$

\begin{abstract}
RESUMEN
El tromboembolismo pulmonar es la manifestación más grave de la enfermedad tromboembólica venosa y la tercera causa de mortalidad cardiovascular. Su sintomatología puede ir desde un cuadro asintomático o con síntomas leves, hasta el paro cardiaco. Dentro del enfoque de esta patología es importante tener en cuenta escalas que permiten estimar la probabilidad de que determinado paciente con ciertos signos, síntomas y factores de riesgo presente un tromboembolismo pulmonar, así como escalas diseñadas para valorar el riesgo de morir, en pacientes en los que ya se estableció este diagnóstico. Los pilares del tratamiento son la anticoagulación y la trombólisis, sin embargo, esta última está indicada únicamente en algunos casos. La presente revisión tiene como objetivo presentar una actualización de la evidencia sobre el enfoque diagnóstico y terapéutico del tromboembolismo pulmonar agudo, desde el ingreso del paciente al servicio de urgencias.
\end{abstract}

Palabras clave: tromboembolia venosa; embolia pulmonar; terapia trombolítica; urgencias médicas; tomografía computarizada multidetector.

\section{ABSTRACT}

Pulmonary embolism is the most severe manifestation of venous thromboembolic disease and the third cause of cardiovascular mortality. Its symptoms can range from asymptomatic or mild symptoms to cardiac arrest. The approach to patients with suspected pulmonary embolism includes scores that allow estimating the clinical probability that a certain patient with certain signs, symptoms and risk factors will present a pulmonary thromboembolism, as well as scores that classify patients by severity and risk of hemodynamic decompensation. Treatment is based on anticoagulation and thrombolysis, which is used only in certain patients. The goal of this review is to present updated evidence regarding diagnosis and treatment of acute pulmonary embolism from the moment the patient arrives at the emergency room.

Keywords: venous thromboembolism; pulmonary embolism; thrombolytic therapy; emergencies; multidetector computarized tomography

\section{RESUMO}

A embolia pulmonar é a manifestação mais grave da doença tromboembólica venosa e a terceira principal causa de mortalidade cardiovascular. Seus sintomas podem variar de sintomas assintomáticos ou leves a parada cardíaca. No enfoque desta patologia, é importante levar em consideração escalas que permitem estimar a probabilidade de um determinado paciente com determinados sinais, sintomas e fatores de risco apresentar 
tromboembolismo pulmonar, bem como escalas destinadas a avaliar o risco de morrer, em pacientes nos quais esse diagnóstico já foi estabelecido. Os pilares do tratamento são a anticoagulação e a trombólise, porém, esta última está indicada apenas em alguns casos. A presente revisão tem como objetivo apresentar uma atualização das evidências sobre a abordagem diagnóstica e terapêutica do tromboembolismo pulmonar agudo, desde a admissão do paciente no pronto-socorro.

Palavras-chave: tromboembolismo venoso; embolia pulmonar; terapia trombolítica; emergência Médica; tomografia computadorizada com multidetectores

\section{INTRODUCCIÓN}

El tromboembolismo pulmonar (TEP) es el bloqueo de una arteria pulmonar o una de sus ramas por alguna sustancia que viaja por el torrente sanguíneo desde otro lugar del cuerpo ${ }^{1}$. Es la manifestación más grave del espectro de la enfermedad tromboembólica venosa (TEV), que incluye el TEP y la trombosis venosa profunda (TVP). Es común, con una incidencia de 60 a 112 casos por cada $100000^{2}$, es la tercera causa de mortalidad cardiovascular ${ }^{3}$, después del infarto agudo de miocardio y del accidente cerebrovascular ${ }^{4}$ y causa al menos 300000 muertes al año en Estados Unidos ${ }^{5}$. Puede ser mortal en la fase aguda o conducir a enfermedades crónicas y discapacidad $^{6}$.

Las manifestaciones clínicas son variables, abarcan desde la disnea leve hasta paro cardiorrespiratorio ${ }^{7}$. E1TEP se debe considerar dentro del diagnóstico diferencial de todo paciente con inicio agudo o empeoramiento de su disnea, hipotensión y dolor precordial ${ }^{8}$. Según la sospecha clínica, la evaluación diagnóstica incluye biomarcadores (troponinas y péptido natriurético cerebral o BNP) o requerir imágenes, como la angiografía por tomografía computarizada de tórax (AngioTAC) o una gammagrafía de ventilación perfusión ${ }^{9,10}$. Adicionalmente, la ecocardiografía puede ser de gran utilidad en pacientes en estado de choque, en quienes su condición crítica impide la realización de otros estudios imagenológicos ${ }^{11}$.

Existen múltiples escalas para la estratificación del riesgo, y para establecer el riesgo de complicaciones y de muerte. La clasificación del riesgo determina la disposición del paciente en un servicio concreto (unidad de alta dependencia, hospitalización, manejo ambulatorio) y quiénes son candidatos a trombólisis, sin embargo, existen controversias al respecto 5 .

Los pilares del tratamiento son la anticoagulación y la trombólisis sistémica, que está indicada en pacientes de alto riesgo y, según la relación riesgo-beneficio, en aquellos de riesgo intermedio-alto ${ }^{2,5}$.

El objetivo de esta revisión es describir el enfoque del tromboembolismo pulmonar desde el servicio de urgencias, con la disnea como síntoma cardinal. Se comentan las escalas de probabilidad y riesgo, y se propone un algoritmo basado en preguntas y una exploración de las intervenciones terapéuticas de acuerdo con la evidencia actual.

\section{TEMA CENTRAL}

\section{Enfoque diagnóstico}

\section{1. ¿En qué pacientes debe ser sospechado el TEP?}

El TEP se debe sospechar en todos los pacientes que presentan disnea de novo o aumento progresivo de su disnea, asociado a dolor torácico. Según el estudio EMPEROR (Multicenter Emergency Medicine Pulmonary Embolism in the Real World Registry), los motivos de consulta más comunes en pacientes con TEP confirmado o altamente sospechoso son la disnea en reposo (50\%), dolor pleurítico (39\%), disnea de esfuerzos (27\%) y edema de miembros inferiores sugestivo de TVP $(24 \%)^{12}$.

El paciente puede presentar también hipotensión sostenida sin causa clara, hipoxemia, disociación electromecánica o paro cardiaco, por lo que se debe tener entre los diagnósticos diferenciales en este tipo de $\operatorname{casos}^{13}$.

\section{2. ¿Presenta el paciente factores de riesgo?}

El TEP resulta de una interacción entre los factores de riesgo individuales del paciente, que no son modificables, y las circunstancias clínicas en las que se encuentra (usualmente transitorias), que incluyen la inmovilización, el trauma o la cirugía mayor. Entre los factores de riesgo del individuo se encuentran la edad, antecedente de TEV, malignidad activa, otras comorbilidades como falla cardiaca o respiratoria, alguna trombofilia, embarazo, consumo de anticonceptivos orales o terapia de remplazo hormonal ${ }^{14}$. La presencia de TVP cobra especial importancia, ya que es el desencadenante de un gran número de episodios de TEP y se asocia con un peor pronóstico, por lo que es un predictor de mortalidad por cualquier causa a los 30 días (OR 1.9 IC 95\% 1.5-2.4) ${ }^{15}$.

La mayoría de pacientes con TEP tiene uno o más factores de riesgo (TEP secundario), pero en algunos de ellos (aproximadamente el 30\%) no se logran identificar 
dichos factores (TEP idiopático o no provocado) ${ }^{16}$. La probabilidad clínica de TEP aumenta en proporción al número de factores de riesgo presentes, por lo tanto, es de gran importancia indagar sobre estos ${ }^{17}$.

De la misma manera, existen factores de riesgo que predisponen a TEP recurrente, a pesar de recibir anticoagulación apropiada. Entre ellos están la inmovilización, el cáncer, la enfermedad pulmonar obstructiva crónica, el sexo masculino, la obesidad, el colesterol de alta densidad (HDL) menor de $40 \mathrm{mg} / \mathrm{dl}$ y la falta de recanalización de una TVP en un doppler venoso de seguimiento ${ }^{14}$.

\section{3. ¿Cuál es la presentación clínica?}

Los signos y síntomas clínicos del TEP agudo son muy inespecíficos. ElTEP puede ser asintomático como diagnóstico incidental, hasta llegar a tener clínica de disnea súbita, dolor torácico, síncope, hemoptisis o inestabilidad hemodinámica ${ }^{18}$. De acuerdo con los resultados de un estudio reciente, el TEP agudo es frecuente en pacientes con síncope (17\%), por lo tanto, siempre debe ser tenido en cuenta como un diagnóstico diferencial ${ }^{19}$.

Los síntomas varían según la localización del trombo en la circulación pulmonar, visualizado mediante angioTAC. Se llama TEP central a los trombos en el tronco principal de la arteria pulmonar o en la arteria pulmonar principal derecha o izquierda. Se considera que el TEP es periférico cuando el trombo está alojado en alguna arteria pulmonar segmentaria o subsegmentaria ${ }^{20}$. En el TEP central la disnea puede ser aguda y grave, en TEP periférico suele ser leve e incluso transitoria. La presentación es tan variada que grandes trombos pueden ser asintomáticos al inicio y tener un desenlace fatal, o en el otro extremo, los émbolos pequeños pueden ser muy sintomáticos, especialmente en el contexto de una baja reserva cardiovascular ${ }^{5,18}$.

El dolor torácico es un síntoma frecuente de TEP, es causado por irritación de la pleura debido a embolismos distales que causan infarto pulmonar ${ }^{21}$. En el TEP central el dolor en el pecho tiene características típicas de angina. Es por esto por lo que se debe tener en cuenta el síndrome coronario agudo o la disección aórtica dentro del diagnóstico diferencial ${ }^{18}$. La disminución de la saturación arterial de oxígeno $(\mathrm{SaO} 2)$ es frecuente, menos del $40 \%$ de los pacientes tiene $\mathrm{SaO} 2$ normal ${ }^{22,23}$.

Los sindromes clásicos de presentación de TEP y su frecuencia, según el estudio PIOPED II son ${ }^{24}$ :

A. Síndrome de hemorragia o infarto pulmonar, caracterizado por hemoptisis o dolor pleurítico (41\%): ocurre con más frecuencia en pacientes con infarto pulmonar y se determina por émbolos más pequeños y periféricos, donde es posible auscultar roce pleural.

B. Síndrome de disnea aislada en ausencia de dolor pleurítico, hemoptisis o colapso circulatorio (36\%): se debe a un TEP más central con consecuencias hemodinámicas prominentes, puede haber dolor torácico retroesternal que simula un dolor anginoso. Ocasionalmente, el comienzo de la disnea es progresivo durante semanas y se llega al diagnóstico de TEP por la ausencia de otras causas clásicas de disnea progresiva. En pacientes con insuficiencia cardiaca o enfermedad pulmonar prexistentes, el empeoramiento de la disnea puede ser el único síntoma que indique $\mathrm{TEP}^{5,25}$.

C. Síndrome de colapso circulatorio (5\%): es la forma más grave de presentación del TEP. Se considera que un paciente está cursando con inestabilidad hemodinámica cuando cumple alguna de las siguientes condiciones ${ }^{5}$ : - Paro cardiaco: el ritmo más común es la actividad eléctrica sin pulso (36\%). Se estima que el TEP representa $4.5 \%$ del total de los paros cardiacos.

- Hipotensión persistente: presión arterial sistólica (PAS) $<90 \mathrm{mmHg}$ o caída de más de $40 \mathrm{mmHg}$ con respecto al valor basal por 15 minutos o más, no explicada por sepsis, arritmia o hipovolemia.

- Shock obstructivo: PAS $<90 \mathrm{mmHg}$ o requerimiento de vasopresores o inotrópicos para mantener la PAS por encima de este valor, a pesar de una volemia adecuada, asociado a signos de hipoperfusión (estado mental alterado, piel fría o moteada, oliguria/anuria, aumento del lactato sérico).

\section{Probabilidad clínica de TEP}

Existen escalas que integran la clínica y los factores de riesgo del paciente para establecer qué tan probable es que el cuadro clínico sea explicado por un TEP ${ }^{26}$. Un metaanálisis que comparó la Escala de Ginebra y la Regla de Wells confirmó un rendimiento diagnóstico similar. Por lo tanto, cualquiera de estas es suficiente en un paciente dado. En la Tabla 1 se muestran las variables para tener en cuenta en la Regla de Wells y en la Escala de Ginebra-Revisada, muy usadas en Colombia ${ }^{26,27}$.

Estas escalas clasifican al paciente dentro de dos grupos: probabilidad intermedia/baja (o TEP improbable) y probabilidad alta (o TEP probable). La proporción de pacientes con TEP confirmado es del 10\% en la categoría de baja probabilidad, del $30 \%$ en la de probabilidad moderada y del $65 \%$ en la de probabilidad alta ${ }^{26}$.

Adicionalmente, la escala Pulmonary Embolism Rule Out Criteria (PERC) cuenta con ocho criterios para descartar TEP en pacientes con una probabilidad previamente baja. Tiene una sensibilidad diagnóstica de más del 95\%, por lo que permite excluir TEP en pacientes con probabilidad baja o intermedia ${ }^{28}$. Los estudios han mostrado que el riesgo embólico a los tres meses fue $<1 \%$ en pacientes con probabilidad clínica baja o intermedia con PERC negativo. La escala posibilita descartar TEP cuando se cumplen todos los criterios ${ }^{28}$ : 
Tabla 1. Escalas para evaluar la probabilidad clínica de tromboembolismo pulmonar.

\begin{tabular}{|c|c|c|}
\hline \multirow[b]{2}{*}{ Escala } & \multicolumn{2}{|c|}{ Puntaje } \\
\hline & $\begin{array}{l}\text { Versión } \\
\text { original }\end{array}$ & $\begin{array}{c}\text { Versión } \\
\text { simplificada }\end{array}$ \\
\hline \multicolumn{3}{|l|}{ Wells } \\
\hline Signos clínicos de TVP & 3 & 1 \\
\hline $\begin{array}{l}\text { Diagnóstico alternativo } \\
\text { menos probable que TEP }\end{array}$ & 3 & 1 \\
\hline $\begin{array}{l}\text { Cirugía reciente o } \\
\text { inmovilización }\end{array}$ & 1.5 & 1 \\
\hline Frecuencia cardiaca $>100$ & 1.5 & 1 \\
\hline Antecedente de TEP o TVP & 1.5 & 1 \\
\hline Hemoptisis & 1 & 1 \\
\hline Malignidad & 1 & 1 \\
\hline Probabilidad baja & $<2$ & \\
\hline Probabilidad moderada & $2-6$ & \\
\hline Probabilidad alta & $>6$ & \\
\hline TEP improbable & $0-4$ & $\leq 1$ \\
\hline TEP probable & $\geq 5$ & $>1$ \\
\hline \multicolumn{3}{|l|}{ Ginebra (revisada) } \\
\hline Frecuencia cardiaca $\geq 95$ & 5 & 2 \\
\hline Frecuencia cardiaca 75-94 & 3 & 1 \\
\hline Antecedente de TVP o TEP & 3 & 1 \\
\hline $\begin{array}{l}\text { Dolor unilateral en } \\
\text { miembro inferior }\end{array}$ & 3 & 1 \\
\hline $\begin{array}{l}\text { Dolor a la palpación en } \\
\text { miembro inferior y edema } \\
\text { unilateral }\end{array}$ & 4 & 1 \\
\hline $\begin{array}{l}\text { Cirugía por fractura en el } \\
\text { último mes }\end{array}$ & 2 & 1 \\
\hline Hemoptisis & 2 & 1 \\
\hline Cáncer activo & 2 & 1 \\
\hline Edad > 65 años & 1 & 1 \\
\hline Probabilidad baja & $0-3$ & $0-1$ \\
\hline Probabilidad intermedia & $4-10$ & $2-4$ \\
\hline Probabilidad alta & $\geq 11$ & $\geq 5$ \\
\hline TEP improbable & $0-5$ & $0-2$ \\
\hline TEP probable & $\geq 6$ & $\geq 3$ \\
\hline
\end{tabular}

- Edad <50 años

- Frecuencia cardiaca $<100$ latidos por minuto

- $\mathrm{SaO} 2$ ambiente $\geq 95 \%$

- No hemoptisis

- No uso de estrógenos endógenos

- No TVP o TEP previo

- No cirugía o trauma que requiera hospitalización en las últimas cuatro semanas

- No edema unilateral de miembro inferior

\section{5. ¿Qué ayuda diagnóstica se debe utilizar?}

Las ayudas diagnósticas señaladas a continuación contribuyen a confirmar la sospecha clínica de TEP o a descartar otros diagnósticos. Es importante aclarar que se solicitan de acuerdo con la probabilidad clínica, la gravedad del cuadro y la estabilidad hemodinámica del paciente $^{5}$.

Dímero D: indicado en pacientes con probabilidad clínica baja/intermedia, ya que su valor predictivo negativo es alto y un resultado en límites normales hace que el diagnóstico de TEP sea improbable. En el contexto de urgencias, un dímero $\mathrm{D}$ negativo sumado a probabilidad intermedia o baja de TEP excluye la enfermedad, sin necesidad de realizar más pruebas, en aproximadamente $30 \%$ de los pacientes ${ }^{29,30}$.

En general, el límite superior de la normalidad de los valores de dímero $\mathrm{D}$ es $500 \mathrm{mg} / \mathrm{dl}$, pero para pacientes mayores de 50 años es preferible utilizar valores ajustados para la edad, para lo que se multiplica la edad del paciente por 10. Cuando se ajusta el valor de corte por edad aumenta el porcentaje de pacientes en los que se excluye el TEP, del 6.4 al 30\% ${ }^{31}$.

Al ser un producto de la degradación de la fibrina, el dímero $\mathrm{D}$ se encuentra elevado en muchos otros contextos, lo que hace que su especificidad sea baja. Algunos ejemplos son los pacientes sometidos a venopunción, hospitalizados, con cáncer, con enfermedades infecciosas o inflamatorias y en embarazo ${ }^{32,33}$. En consecuencia, el valor predictivo positivo de un dímero $\mathrm{D}$ elevado es bajo y su determinación no es útil para confirmar el TEP en pacientes que tienen alta probabilidad clínica ${ }^{30}$.

Electrocardiograma: los cambios electrocardiográficos dejan sospechar TEP, pero no confirman el diagnóstico, ya que su sensibilidad y especificidad son limitadas. Dentro de los hallazgos se encuentran la inversión de las ondas T en las derivaciones V1-V4, el patrón QR en V1, con ondas Rs prominentes en $\mathrm{V} 1$ y V2 y bloqueo incompleto o completo de la rama derecha del haz de His. El patrón S1Q3T3 se caracteriza por ondas S en DI, ondas Qen DIII y ondas T invertidas en DIII, se ha descrito clásicamente en TEP, aunque su sensibilidad es solo del $54 \%$ y su especificidad del $62 \%$. En casos leves, la única anomalía puede ser la taquicardia sinusal, el hallazgo más sensible, que está presente hasta en un $40 \%$ de los pacientes. Finalmente, puede haber arritmias auriculares, donde la más común es la fibrilación auricular ${ }^{5}$.

Radiografía de tórax: es útil especialmente para excluir otras causas de disnea o dolor torácico. Se han descrito varios hallazgos radiográficos que sugieren TEP, sin embargo, son poco sensibles, por lo que una radiografía de tórax sin alteraciones no descarta el diagnóstico ${ }^{34}$ :

- Signo de Westermark: relacionado con la oligoemia o la hipoperfusión pulmonar distal al trombo. A pesar de 
que es poco frecuente ( $2-6 \%$ de pacientes), es uno de los más específicos.

- Signo de Fleischner: aumento de tamaño de la arteria pulmonar afectada y amputación de esta.

- Atelectasias laminares: aparecen en el 50\% de los casos.

- Consolidación multifocal de predominio inferior.

- Joroba de Hampton: opacidad triangular de base pleural y vértice hacia el hilio, muy poco frecuente.

Marcadores de disfunción ventricular y lesión miocárdica: la determinación de biomarcadores de laboratorio, como troponinas cardiacas y BNP, hace parte de la estratificación del riesgo de los pacientes con TEP ya diagnosticado. El resultado positivo de uno de estos biomarcadores, asociado a un Pulmonary Embolism Severity Index (PESI) simplificado $>1$, implica riesgo intermedio de mortalidad temprana 5 .

Ecocardiograma: el TEP agudo produce sobrecarga por presión y lleva a disfunción del ventrículo derecho, detectable mediante ecocardiografía. Los hallazgos más descritos son TAPSE < $16 \mathrm{~mm}$, razón del diámetro del ventrículo derecho con respecto al izquierdo mayor o igual a uno e hipocinesia de pared libre (signo de McConnell) $)^{5,9,35}$. Ante la sospecha de TEP en un paciente con inestabilidad hemodinámica, que no pueda ser llevado a angio TAC, la ausencia de signos ecocardiográficos de sobrecarga o de disfunción del ventrículo derecho prácticamente excluye el TEP. Por otro lado, el ecocardiograma es de utilidad en la estratificación de riesgo de los pacientes estables, ya que ayuda a diferenciar entre riesgo intermedio-alto, intermedio-bajo o bajo ${ }^{5}$.

AngioTAC: la angioTAC con multidetectores permite ver las arterias pulmonares hasta el nivel subsegmentario y es la prueba de referencia para el diagnóstico de TEP. Es la imagen de primera línea para pacientes con probabilidad clínica alta y está indicado también en pacientes con probabilidad intermedia o baja con dímero D elevado ${ }^{5}$. Su sensibilidad es del $83 \%$ al $96 \%$ y su valor predictivo positivo es mayor en pacientes con probabilidad clínica alta ${ }^{24}$.

Gammagrafía de Ventilación/Perfusión(V/Q): es una opción para los pacientes con dímero $\mathrm{D}$ alto que tengan alguna contraindicación para la angioTAC. Puede ser de elección en mujeres jóvenes para evitar radiación y disminuir el riesgo de cáncer de mama. El número de individuos con hallazgos no concluyentes disminuye si se tiene en cuenta la probabilidad clínica. Por lo tanto, los pacientes con una gammagrafía pulmonar no diagnóstica y baja probabilidad clínica de TEP tienen prevalencia baja de TEP confirmado ${ }^{24}$. Cuando la gammagrafía V/Q muestra probabilidad alta en un paciente con baja probabilidad clínica de TEP, se debe considerar la confirmación mediante otras pruebas 5 .
Detección de TVP en miembros inferiores: en un gran número de pacientes el TEP se origina a partir de una TVP en una extremidad inferior, por tanto, es importante tener en cuenta esta posibilidad durante la anamnesis y el examen físico ${ }^{5}$. La ecografía de compresión tiene sensibilidad $>90 \%$ y especificidad de $95 \%$ para TVP sintomática ${ }^{36,37}$. Detecta TVP en un 30-50\% de los pacientes con $\mathrm{TEP}^{7,36}$, y el hallazgo de TVP proximal en pacientes con sospecha de TEP se considera suficiente para iniciar anticoagulante sin pruebas diagnósticas adicionales ${ }^{38}$. El único criterio diagnóstico validado para la TVP es la compresibilidad incompleta de la vena, que indica la presencia de un coágulo, mientras que las mediciones de flujo no son fiables 5 .

\section{Escalas de riesgo}

Una vez establecido el diagnóstico de TEP, el siguiente paso es estratificar los pacientes según su riesgo de morir, ya que esto es fundamental para determinar el enfoque terapéutico ${ }^{5,39}$.

En primer lugar, es necesario identificar pacientes con inestabilidad hemodinámica. Estos corresponden a la categoría de alto riesgo, en ellos no es necesario realizar ayudas diagnósticas ni escalas adicionales para definir la conducta terapéutica, ya que son casos críticos que deben ser vigilados en unidades de alta dependencia y cumplen con la indicación de trombólisis ${ }^{5,40}$.

En la estratificación del riesgo de los pacientes que no cursan con inestabilidad hemodinámica es necesario incluir algún índice de gravedad, que tenga en cuenta la clínica y los factores de riesgo, así como parámetros paraclínicos (BNP y troponinas) e imagenológicos (ecocardiografía transtorácica o angioTAC) que permitan evaluar la disfunción o necrosis ventricular derecha ${ }^{5}$.

El Pulmonary Embolism Severity Index (PESI) es una escala ampliamente validada que valora el riesgo de mortalidad a 30 días, según parámetros clínicos ${ }^{18}$. Incluye factores como edad, sexo, antecedente de cáncer, falla cardiaca crónica, EPOC, frecuencia cardiaca (FC) mayor a 110, PAS menor de $100 \mathrm{mmHg}$, frecuencia respiratoria mayor a 30 , temperatura menor a $36^{\circ} \mathrm{C}$, estado mental alterado y $\mathrm{SaO} 2$ menor de $90 \%$. Su principal ventaja radica en la identificación confiable de pacientes con bajo riesgo de morir (clases PESI I y II). Las clases PESI III, IV y $\mathrm{V}$ corresponden a una mortalidad moderada, alta y muy alta ${ }^{5}$.

Recientemente se ha desarrollado y validado una versión simplificada del PESI (sPESI) ${ }^{41}$, que cuenta con un menor número de variables, donde cada una suma un punto. Tiene en cuenta la edad mayor de 80 años, antecedente de cáncer, falla cardiaca o EPOC, FC mayor o igual a 110, PAS menor de $100 \mathrm{mmHg}$ y $\mathrm{SaO} 2<90 \%$. 
Se considera que un sPESI de 0 puntos es equivalente a las clases I y II del PESI, y un resultado mayor o igual a uno corresponde a un PESI III - $\mathrm{V}^{5,41}$.

Teniendo en cuenta lo anterior, los pacientes de bajo riesgo son aquellos que pertenecen a la categoría I o II del PESI y que no tienen aumento de troponinas ni evidencia ecocardiográfica de disfunción ventricular derecha. Se clasifican dentro del riesgo intermedio aquellos PESI III a V que, a su vez se subdivide en riesgo intermedio-alto, cuando hay evidencia de disfunción del ventrículo derecho por ecocardiografía/TAC y aumento de troponinas/BNP, y en riesgo intermedio-bajo, cuando solo una o ninguna de las pruebas anteriores es positiva ${ }^{5}$.

En resumen, los pacientes con TEP se dividen en los siguientes grupos según su riesgo de fallecer, ${ }^{5,9}$ :

- TEP masivo o de alto riesgo: inestabilidad hemodinámica.

- TEP submasivo o de riesgo intermedio:

- a. Intermedio-alto: PESI III-V + evidencia imagenológica de disfunción ventricular derecha + aumento de marcadores cardiacos.

- b. Intermedio-bajo: PESI III-V + una (o ninguna) de las anteriores.

- TEP de bajo riesgo: PESI I-II, ausencia de signos de disfunción ventricular derecha o aumento de marcadores cardiacos.

Los pacientes con riesgo bajo e intermedio-bajo se benefician únicamente de anticoagulación. En aquellos con riesgo intermedio, la trombólisis es controversial. En el grupo de riesgo intermedio-alto se podría considerar la trombólisis según la relación riesgo-beneficio ${ }^{5}$, sin embargo, varios estudios como el Pulmonary Embolism International Thrombolysis Trial (PEITHO), no han demostrado beneficio sobre la mortalidad a siete o 30 días, pero sí un aumento significativo del riesgo de sangrado ${ }^{42}$. En el grupo de riesgo alto está indicada la trombólisis, ya que los beneficios superan los riesgos ${ }^{40,43}$.

\section{Enfoque terapéutico}

\section{Terapia anticoagulante}

El tratamiento del TEP se inicia con la administración de anticoagulantes, que deben ser administrados en todos los pacientes con el diagnóstico, independiente de su estratificación de riesgo. Estos agentes han demostrado prevenir la recurrencia de los síntomas y la muerte temprana $a^{7,43}$.

La meta de la anticoagulación inicial es inhibir la formación de trombos adicionales 9 . Puede llevarse a cabo con heparina no fraccionada (HNF) intravenosa (IV), heparinas de bajo peso molecular (HBPM) o fondaparinux subcutánea (SC), durante los primeros 10 días $^{7,44,45}$. La dosis inicial de HNF es $80 \mathrm{UI} / \mathrm{kg}$, seguida de $18 \mathrm{UI} /$ $\mathrm{kg}$ /hora, ajustada según el TPT. Las dosis de la HBPM y del fondaparinux también se ajustan según el peso y se administran dos veces al día ${ }^{43}$.

Después de estabilizar al paciente, la anticoagulación parenteral se sustituye con antagonistas de la vitamina $\mathrm{K}$ (warfarina) o con anticoagulantes orales directos (DOACs) como apixaban, rivaroxaban, dabigatran y endoxaban (no disponible en Colombia) ${ }^{5,74}$. Los pacientes que toman warfarina deben recibir terapia puente con anticoagulación parenteral, hasta lograr un INR (international normalized ratio) entre dos y cuatro, por al menos dos días consecutivos ${ }^{7}$. Los DOACs se inician inmediatamente o dos días después de la anticoagulación parenteral ${ }^{7}$.

Los anticoagulantes son administrados por un mínimo de tres meses o de manera indefinida, dependiendo del origen del trombo ${ }^{5,43,46}$.

\section{Trombólisis}

Los trombolíticos convierten plasminógeno en plasmina, que hidroliza la fibrina del trombo, lo que conduce a la lisis del coágulo ${ }^{44}$. La estreptoquinasa, La urokinasa (también conocida como activador del plasminógeno urinario) y la alteplasa son los únicos agentes aprobados para $\mathrm{TEP}^{47,48}$. Los trombolíticos de tercera generación

Tabla 2. Comparación de los agentes trombolíticos, que incluye si su uso está aprobado en tromboembolismo pulmonar.

\begin{tabular}{lccccc} 
& Estreptokinasa & Urokinasa & Alteplasa & Reteplasa & \multicolumn{2}{c}{ Tenecteplasa } \\
\hline Generación & $1 a$ & $1 a$ & $2 a$ & $3 a$ & $4^{a}$ \\
\hline Fibrina-específico & No & No & Sí & Sí & Sí \\
\hline Vida 1/2, horas & 12 & $7-20$ & $4-10$ & $11-19$ & $15-24$ \\
\hline Aprobación FDA & Sí & Sí & Sí & No & No \\
\hline
\end{tabular}


como el tenecteplase y el reteplase están aprobados para el síndrome coronario agudo, pero por el momento no se incluyen en las guías de TEP, a pesar de que existen estudios $^{49,50}$. El alteplase, el reteplase y el tenecteplase activan el plasminógeno en la superficie del coágulo por lo que son fibrina-específicos ${ }^{51}$. Estos últimos tienen vidas medias más largas, lo que permite su administración en bolo, y conllevan riesgo menor de reacciones alérgicas, más asociadas a los trombolíticos de primera generación ${ }^{51}$. Los trombolíticos de primera generación activan el plasminógeno de forma más generalizada y no solo el que está presente en la matriz del coágulo, lo que favorece mayor sangrado ${ }^{49}$. En la Tabla 2 se resumen las características de los diferentes trombolíticos, respecto a su clasificación y vida media ${ }^{2}$.

La terapia trombolítica mejora la presión de la arteria pulmonar, la oxigenación arterio-venosa, la perfusión pulmonar y los hallazgos ecocardiográficos, además disminuye los síntomas clínicos, previene el TEP recurrente y reduce la mortalidad ${ }^{19}$. Sin embargo, estos beneficios pueden llegar a no ser mayores que el riesgo de sangrado ${ }^{43}$; no existe una herramienta validada para predecir este riesgo en pacientes sometidos a trombólisis, por lo que se deben tener en cuenta los factores de riesgo identificados, como enfermedades cerebrovasculares, tanto hemorrágicas como isquémicas, trauma o cirugía en las últimas tres semanas o anticoagulación previa ${ }^{5,40}$.

\section{Tratamiento según la clasificación de riesgo}

\section{TEP de alto riesgo}

Además de la anticoagulación y la estabilización hemodinámica, la principal medida terapéutica de este grupo de pacientes es la trombólisis. La Sociedad Europea de Cardiología (ESC) clasifica como una recomendación 1B la administración de terapia trombolítica en pacientes con TEP agudo de alto riesgo y las guías CHEST actualizadas en 2016, como una recomendación de grado $2 \mathrm{~B}^{11,46,48}$.

En el contexto del TEP de alto riesgo, los beneficios de la trombólisis sistémica superan los riesgos, por lo que solo debe evitarse en presencia de hemorragia activa e incontrolable ${ }^{7,39}$. Se han estudiado diferentes dosis y duraciones de la infusión. Las pautas de la ESC recomiendan un régimen de alteplasa de $100 \mathrm{mg}$ en infusión durante dos horas, en lugar de trombolíticos de primera generación, que requieren una infusión más prolonga$\mathrm{da}^{5,11}$. Los trombolíticos proporcionan mayor beneficio, si se administran dentro de las 48 horas del inicio de los síntomas ${ }^{7}$. En pacientes con contraindicaciones para la terapia trombolítica puede considerarse la trombectomía mecánica ${ }^{22}$.

\section{TEP de riesgo intermedio}

El papel de la trombólisis en pacientes hemodinámicamente estables con TEP submasiva sigue siendo tema de discusión. Se deben tener en cuenta los riesgos y beneficios de la terapia trombolítica en cada paciente, así como la estratificación del paciente según sus comorbilidades y riesgo de morir ${ }^{40,52}$.

Pacientes con TEP de riesgo intermedio-alto, que luego experimentan deterioro hemodinámico súbito, pueden ser considerados para terapia trombolítica, ${ }^{5,39}$. Se han realizado múltiples estudios que buscan evaluar el tratamiento trombolítico en pacientes con TEP de riesgo intermedio.

El estudio MAPPET- $3^{49}$ (2002) fue uno de los primeros que evaluó los trombolíticos en estos pacientes. Este comparó el tratamiento con $100 \mathrm{mg}$ de alteplasa más heparina, versus heparina más placebo. El objetivo primario fue evaluar la incidencia de muertes intrahospitalarias o el deterioro clínico de pacientes que requerirían escalar el tratamiento a terapia trombolítica. $\mathrm{La}$ incidencia del desenlace primario fue mayor en el grupo de heparina más placebo, que en el grupo de heparina más alteplasa $(\mathrm{p}=0.006)$, y la probabilidad de supervivencia sin eventos a los 30 días fue mayor en el grupo de heparina más alteplasa ( $\mathrm{p}=0.005)$. Sin embargo, no se observaron diferencias en las muertes hospitalarias $(p=0,71)^{47}$. Las complicaciones hemorrágicas asociadas con la alteplasa dependen de la dosis, por lo que se ha planteado si la dosis de $100 \mathrm{mg}$ en infusión durante dos horas es apropiada para todos los pacientes con TEP ${ }^{50}$. Después, los estudios PEITHO y MOPETT utilizaron dosis más bajas de trombolíticos según el peso.

E1 PEITHO fue un ensayo aleatorizado, doble ciego, que comparó tenecteplase más heparina versus heparina más placebo. Se incluyeron 1005 pacientes con disfunción ventricular derecha. Se administró una dosis de tenecteplasa de $50 \mathrm{mg}$ en pacientes con peso mayor a $90 \mathrm{~kg}$ y $30 \mathrm{mg}$ en pacientes con peso menor a $60 \mathrm{~kg}^{42}$. $\mathrm{El}$ resultado primario del estudio fue la muerte o la descompensación hemodinámica dentro de los siete días siguientes, lo que ocurrió en el 2.6\% de los pacientes en el grupo de tenecteplasa, en comparación con $5.6 \%$ en el grupo placebo $(p=0.02)$. Los eventos adversos fueron: hemorragia extracraneal en el 6.3\% del grupo de tenecteplasa y en $1.2 \%$ en el grupo placebo ( $p<0.001$ ) y accidente cerebrovascular hemorrágico en $2.4 \%$ en el grupo de tenecteplasa, versus $0.2 \%$ en el grupo placebo $(\mathrm{p}=0.003)$. Por tanto, se demostró que la terapia trombolítica previene la descompensación hemodinámica, con un mayor riesgo de hemorragia grave y accidente cerebrovascular ${ }^{42}$.

Un análisis post hoc del estudio PEITHO sugirió que dos o más de los siguientes criterios implican mayor riesgo de complicaciones mayores: presión arterial sistólica 
$\leq 110 \mathrm{mmHg}$, frecuencia respiratoria $>20$ respiraciones por minuto, antecedentes de insuficiencia cardiaca y cáncer activo $^{43}$.

Según la evidencia actual, la indicación de trombólisis en TEP de riesgo intermedio-alto debe evaluar el beneficio, teniendo en cuenta la edad, la clase funcional, las comorbilidades y la gravedad clínica; se debe sopesar el riesgo de sangrado.

\section{TEP de bajo riesgo}

Los pacientes con TEP sin inestabilidad hemodinámica o signos de disfunción cardiaca tienen un riesgo bajo de morir a los 30 días $^{47}$. La terapia trombolítica no se recomienda, por lo que estos pacientes se benefician únicamente de anticoagulación. Durante la fase aguda se puede considerar la hospitalización en planta o incluso el alta temprana, si no hay otra razón para el ingreso y si tienen buen soporte social y fácil acceso a servicios de salud 5 .

\section{CONCLUSIONES}

E1TEP es una importante causa de morbimortalidad y un motivo de consulta frecuente en los servicios de urgen- cias. Se debe establecer la probabilidad del diagnóstico de TEP, según los hallazgos clínicos y factores de riesgo, para definir las ayudas diagnósticas necesarias. Una vez se confirme el diagnóstico, es necesario estratificar el riesgo de los pacientes según su estabilidad hemodinámica, índices que reúnen características, valoración clínica (PESI o sPESI) y presencia de disfunción o necrosis ventricular derecha, de acuerdo con biomarcadores cardiacos e imágenes.

El tratamiento anticoagulante es un pilar del tratamiento y debe iniciarse rápidamente en todos los pacientes. Aquellos con inestabilidad hemodinámica, es decir, TEP de alto riesgo, serán monitoreados en unidades de alta dependencia y deben recibir terapia trombolítica, ya que los beneficios superan los riesgos. En pacientes con TEP de riesgo intermedio es controversial el uso de trombólisis, esta se debe considerar cuando el riesgo es intermedio-alto, según las comorbilidades, la clase funcional, los hallazgos clínicos y el riesgo de sangrado.

\section{Declaración de conflictos de intereses}

Los autores declaran no tener ningún conflicto de interés.

\section{REFERENCIAS}

1. Lambrini K, Konstantinos K, Christos I, Petros O, Areti T. Pulmonary embolism: A literature review. Am J Nurs Sci 2017;7:57-66.

2. Marti C, John G, Konstantinides S, Combescure C, Sánchez O, Lankeit M, et al. Systemic thrombolytic therapy for acute pulmonary embolism: A systematic review and meta-analysis. Eur Heart J 2015;36:605-14.

3. Xu Q, Huang $K$, Zhai Z, Yang Y, Wang J, Wang C. Initial thrombolysis treatment compared with anticoagulation for acute intermediate-risk pulmonary embolism: A meta-analysis. J Thorac Dis 2015;7:810-21.

4. Raskob G. Thrombosis: A major contributor to global disease burden. Thromb Haemost 2014;112:843-52.

5. Konstantinides SV, Meyer G, Becattini C, Bueno H, Geersing G-J, Harjola V-P, et al. 2019 ESC Guidelines for the diagnosis and management of acute pulmonary embolism developed in collaboration with the European Respiratory Society (ERS)The Task Force for the diagnosis and management of acute pulmonary embolism of the European Society of Cardiology (ESC). Eur Heart J. 2020;41:543-603.

6. Brækkan SK, Grosse SD, Okoroh EM, Tsai J, Cannegieter SC, Næss IA, et al. Venous thromboembolism, and subsequent permanent work-related disability. J Thromb Haemost 2016;14:1978-87.

7. Righini M, Gal GL, Aujesky D, Roy P-M, Sánchez O, Verschuren F, et al. Diagnosis of pulmonary embolism by multidetector $C T$ alone or combined with venous ultrasonography of the leg: $a$ randomised non-inferiority trial. Lancet 2008;371:1343-52.

8. Wells PS, Ginsberg JS, Anderson DR, Kearon C, Gent M, Turpie AG, et al. Use of a clinical model for safe management of patients with suspected pulmonary embolism. Ann Intern Med 1998;129:997-1005.

9. Jaff MR, McMurtry MS, Archer SL, Cushman M, Goldenberg N, Goldhaber Z., et al. Management of massive and submassive pulmonary embolism, iliofemoral deep vein thrombosis, and chronic thromboembolic pulmonary hypertension. Circulation 2011;123:1788-830.

10. Le Gal G, Righini M, Roy P-M, Sánchez O, Aujesky D, Bounameaux H, et al. Prediction of pulmonary embolism in the emergency department: The Revised Geneva Score. Ann Intern Med 2006;144:165-71. 
11. Kearon C, AkI EA, Comerota AJ, Prandoni P, Bounameaux H, Goldhaber SZ, et al. Antithrombotic therapy for VTE disease. Chest 2012;141:e419S-96S.

12. Pollack CV, Schreiber D, Goldhaber SZ, Slattery D, Fanikos J, O'Neil BJ, et al. Clinical characteristics, management, and outcomes of patients diagnosed with acute pulmonary embolism in the emergency department: Initial report of EMPEROR (Multicenter Emergency Medicine Pulmonary Embolism in the Real-World Registry). J Am Coll Cardiol 2011;57:700-6.

13. Tapson VF. Acute pulmonary embolism. N Engl J Med 2009;358:1037-52.

14. Goldhaber SZ. Risk factors for venous thromboembolism. J Am Coll Cardiol 2010;56:1-7.

15. Becattini C, Cohen AT, Agnelli G, Howard L, Castejón B, Trujillo-Santos J, et al. Risk stratification of patients with acute symptomatic pulmonary embolism based on presence or absence of lower extremity DVT: Systematic review and meta-analysis. Chest 2016;149:192-200.

16. Goldhaber SZ, Visani L, Rosa MD. Acute pulmonary embolism: Clinical outcomes in the International Cooperative Pulmonary Embolism Registry (ICOPER). Lancet 1999;353:1386-9.

17. White $H$. The epidemiology of venous thromboembolism. Circulation 2003;107:4.

18. Donzé J, Gal GL, Fine MJ, Roy P-M, Sánchez O, Verschuren F, et al. Prospective validation of the Pulmonary Embolism Severity Index. Thromb Haemost 2008;100:943-8.

19. Prandoni $P$, Lensing AWA, Prins MH, Ciammaichella M, Perlati M, Mumoli N, et al. Prevalence of pulmonary embolism among patients hospitalized for syncope. N Engl J Med 2016;375:1524-31.

20. Martínez JLA, Sánchez FJA, Echezarreta MAU, García IV, Álvaro JR. Central versus peripheral pulmonary embolism: Analysis of the impact on the physiological parameters and long-term survival. North Am J Med Sci 2016;8:134-42.

21. Stein PD, Henry JW. Clinical characteristics of patients with acute pulmonary embolism stratified according to their presenting syndromes. Chest 1997;112:974-9.

22. Stein PD, Goldhaber SZ, Henry JW, Miller AC. Arterial blood gas analysis in the assessment of suspected acute pulmonary embolism. Chest 1996;109:78-81.

23. Rodger MA, Carrier M, Jones GN, Rasuli P, Raymond F, Djunaedi $H$, et al. Diagnostic value of arterial blood gas measurement in suspected pulmonary embolism. Am J Respir Crit Care Med 2000;162:2105-8.

24. The PIOPED Investigators. Value of the ventilation/perfusion scan in acute pulmonary embolism: Results of the prospective investigation of pulmonary embolism diagnosis (PIOPED). JAMA 1990;263:2753-9.

25. Perrier A, Roy P-M, Aujesky D, Chagnon I, Howarth N, Gourdier A-L, et al. Diagnosing pulmonary embolism in outpatients with clinical assessment, D-Dimer measurement, venous ultrasound, and helical computed tomography: A multicenter management study. Am J Med 2004;116:291-9.

26. Ceriani E, Combescure C, Gal GL, Nendaz M, Perneger T, Bounameaux H, et al. Clinical prediction rules for pulmonary embolism: A systematic review and meta-analysis. J Thromb Haemost 2010;8:957-70

27. Douma RA, Mos ICM, Erkens PMG, Nizet TAC, Durian MF, Hovens MM, et al. Performance of 4 clinical decision rules in the diagnostic management of acute pulmonary embolism. Ann Intern Med 2011;154:709-18.

28. Penaloza A, Soulié C, Moumneh T, Delmez Q, Ghuysen A, Kouri DE, et al. Pulmonary embolism rule-out criteria (PERC) rule in European patients with low implicit clinical probability (PERCEPIC): A multicentre, prospective, observational study. Lancet Haematol 2017;4:e615-21.

29. Perrier A, Roy P-M, Sánchez O, Le Gal G, Meyer G, Gourdier A-L, et al. Multidetector-row computed tomography in suspected pulmonary embolism. N Engl J Med 2005;352:1760-8.

30. Wells PS, Anderson DR, Rodger M, Stiell I, Dreyer JF, Barnes D, et al. Excluding pulmonary embolism at the bedside without diagnostic imaging: Management of patients with suspected pulmonary embolism presenting to the emergency department by using a simple clinical model and D-dimer. Ann Intern Med 2001;135:98-107.

31. Righini M, Van Es J, Den Exter PL, Roy P-M, Verschuren F, Ghuysen A, et al. Age-adjusted D-dimer cutoff levels to rule out pulmonary embolism: The ADJUST-PE Study. JAMA 2014;311:1117-24.

32. Francalanci I, Comeglio P, Liotta AA, Cellai AP, Fedi S, Parretti E, et al. D-dimer concentrations during normal pregnancy, as measured by ELISA. Thromb Res 1995;78:399-405.

33. Righini M, Gal GL, de Lucía S, Roy P-M, Meyer G, Aujesky D, et al. Clinical usefulness of D-dimer testing in cancer patients with suspected pulmonary embolism. Thromb Haemost 2006;95:715-9.

34. Elliott CG, Goldhaber SZ, Visani L, DeRosa M. Chest radiographs in acute pulmonary embolism: Results from the international cooperative pulmonary embolism registry. Chest 2000;118:33-8.

35. Sosland RP, Gupta K. McConnell's Sign. Circulation 2008;118:e517-8.

36. Perrier A, Bounameaux $\mathrm{H}$. Ultrasonography of leg veins in patients suspected of having pulmonary embolism. Ann Intern Med 1998;128:243.

37. Kearon C, Ginsberg JS, Hirsh J. The role of venous ultrasonography in the diagnosis of suspected deep venous thrombosis and pulmonary embolism. Ann Intern Med 1998;129:1044-9.

38. Gal GL, Righini M, Sánchez O, Roy P-M, Baba-Ahmed M, Perrier A, et al. A positive compression ultrasonography of the lower limb veins is highly predictive of pulmonary embolism on computed tomography in suspected patients. Thromb Haemost 2006;95:963-6.

39. Meyer G, Vieillard-Baron A, Planquette B. Recent advances in the management of pulmonary embolism: Focus on the critically ill patients. Ann Intensive Care 2016;6:19. 
40. Martin C, Sobolewski K, Bridgeman P, Boutsikaris D. Systemic thrombolysis for pulmonary embolism: A review. Pharm Ther 2016;41:770-5.

41. Jiménez $D$, Aujesky $D$, Moores L, Gómez V, Lobo JL, Uresandi $F$, et al. Simplification of the Pulmonary Embolism Severity Index for prognostication in patients with acute symptomatic pulmonary embolism. Arch Intern Med 2010;170:1383-9.

42. Meyer G, Vicaut E, Danays T, Agnelli G, Becattini C, Beyer-Westendorf J, et al. Fibrinolysis for patients with intermediate-risk pulmonary embolism. N Engl J Med 2014;370:1402-11.

43. Weinberg I, Jaff MR. Accelerated thrombolysis for pulmonary embolism. Circulation 2014;129:420-1.

44. Sánchez O, Trinquart L, Colombet I, Durieux P, Huisman MV, Chatellier G, et al. Prognostic value of right ventricular dysfunction in patients with haemodynamically stable pulmonary embolism: A systematic review. Eur Heart J 2008;29:1569-77.

45. Janssen Pharmaceuticals, Inc. Xarelto (rivaroxaban) prescribing information [Internet]. 2015 [citado 3 de agosto de 2016]. Disponible en: www.xareltohcp.com/shared/product/xarelto/ prescribing- information.pdf.

46. Collen DC. Molecular mechanisms of fibrinolysis and their application to fibrin-specific thrombolytic therapy. J Cell Biochem 1987;33:77-86.

47. Aujesky D, Obrosky DS, Stone RA, Auble TE, Perrier A, Cornuz J, et al. Derivation and validation of a prognostic model for pulmonary embolism. Am J Respir Crit Care Med 2005;172:1041-6.

48. Kearon C, Akl EA, Ornelas J, Blaivas A, Jiménez D, Bounameaux $\mathrm{H}$, et al. Antithrombotic therapy for VTE disease. Chest 2016;149:315-52.

49. Konstantinides S, Geibel A, Heusel G, Heinrich F, Kasper W. Heparin plus alteplase compared with heparin alone in patients with submassive pulmonary embolism. N Engl J Med 2002;347:114350 .

50. Brandt K, McGinn K, Quedado J. Low-dose systemic alteplase (tPA) for the treatment of pulmonary embolism. Ann Pharmacother 2015;47:818-24.

51. Konstantinides S. Thrombolysis in submassive pulmonary embolism? Yes. J Thromb Haemost 2003;1:1127-9.

52. Vanni S, Nazerian P, Pepe G, Baioni M, Risso M, Grifoni G, et al. Comparison of two prognostic models for acute pulmonary embolism: Clinical vs. right ventricular dysfunction-guided approach. J Thromb Haemost 2011;9:1916-23. 